\title{
Role of kallistatin in prevention of cardiac remodeling after chronic myocardial infarction
}

\author{
Lin Gao, Hang Yin, Robert S Smith Jr, Lee Chao and Julie Chao
}

Oxidative stress causes cardiomyocyte death and subsequent ventricular dysfunction and cardiac remodeling after myocardial infarction (MI), thus contributing to high mortality in chronic heart failure patients. We investigated the effects of kallistatin in cardiac remodeling in a chronic MI rat model and in primary cardiac cells. Human kallistatin gene was injected intramyocardially $20 \mathrm{~min}$ after ligation of the left coronary artery. At 4 weeks after $\mathrm{Ml}$, expression of human kallistatin in rat hearts was identified by reverse transcription-polymerase chain reaction, immunohistochemistry and ELISA. Kallistatin administration improved cardiac performance, increased mean arterial pressure, decreased myocardial infarct size and restored left ventricular wall thickness. Kallistatin treatment significantly attenuated cardiomyocyte size and atrial natriuretic peptide expression. Kallistatin also reduced collagen accumulation, collagen fraction volume and expression of collagen types I and III, transforming growth factor- $\beta 1$ (TGF- $\beta 1$ ) and plasminogen activator inhibitor- 1 in the myocardium. Inhibition of cardiac hypertrophy and fibrosis by kallistatin was associated with increased cardiac nitric oxide (NO) levels and decreased superoxide formation, NADH oxidase activity and p22-phox expression. Moreover, in both primary cultured rat cardiomyocytes and myofibroblasts, recombinant kallistatin inhibited intracellular superoxide formation induced by $\mathrm{H}_{2} \mathrm{O}_{2}$, and the antioxidant effect of kallistatin was abolished by $\mathrm{N} \omega$-nitro-L-arginine methyl ester (L-NAME), indicating a NO-mediated event. Kallistatin promoted survival of cardiomyocytes subjected to $\mathrm{H}_{2} \mathrm{O}_{2}$ treatment, and inhibited $\mathrm{H}_{2} \mathrm{O}_{2}$-induced Akt and ERK phosphorylation, as well as NF- $\kappa$ B activation. Furthermore, kallistatin abrogated TGF- $\beta$-induced collagen synthesis and secretion in cultured myofibroblasts. This is the first study to demonstrate that kallistatin improves cardiac performance and prevents post-MI-induced cardiac hypertrophy and fibrosis through its antioxidant action.

Laboratory Investigation (2008) 88, 1157-1166; doi:10.1038/labinvest.2008.85; published online 1 September 2008

KEYWORDS: kallistatin; cardiac remodeling; hypertrophy; fibrosis; oxidative stress; nitric oxide; TGF- $\beta 1$

Myocardial infarction (MI) leads to cardiac remodeling with complex structural alterations. After an ischemic insult, left ventricle (LV) enlargement is followed by progressive diastolic stiffness, enhanced wall stress and oxygen consumption, thus resulting in cardiomyocyte hypertrophy, interstitial fibrosis and decreased cardiac output. Increased reactive oxygen species (ROS) levels are recognized to be involved in ischemic heart failure and considered to be a major cause of cardiomyocyte death, ventricular dysfunction and heart failure progress. ${ }^{1}$ Under pathological conditions, increased oxidative stress damages DNA and mitochondrial function, activates proapoptotic signaling kinases and leads to myocyte apoptosis or necrosis. ${ }^{2,3}$ Elevation of ROS activates a series of hypertrophic signaling kinases and transcription factors to stimulate cardiac hypertrophy. ${ }^{3,4}$ Moreover, ROS stimulate fibroblast proliferation and activate the expression of profibrotic factors, including transforming growth factor- $\beta 1$ (TGF- $\beta 1$ ), that facilitate fibrosis and matrix remodeling. ${ }^{5-7}$ Elevation of vascular oxidative stress and ventricular remodeling contribute to high mortality in chronic heart failure (CHF) patients. ${ }^{8}$

Kallistatin is a member of the serine proteinase inhibitor (serpin) superfamily and was shown to have pleiotropic effects, including hypotension, anti-angiogenesis and antiinflammation..$^{9-11}$ Kallistatin gene delivery reduced ischemic myocardial inflammation and cardiomyocyte apoptosis at 1 day after acute ischemia/reperfusion injury. ${ }^{12}$ Recently, we observed that increased oxidative stress after MI was associated with reduced circulating kallistatin levels, suggesting that kallistatin might be involved in the progression of 
oxidative cardiac damage. In this study, we investigated the role and mechanisms of kallistatin in cardiac remodeling in a CHF model and in primary cultured cardiomyocytes and myofibroblasts. We found that, after 4 weeks of kallistatin gene therapy, MI-induced heart failure, hypertrophy and fibrosis were greatly improved which were accompanied with inhibition of ROS though NO formation, obstruction of ROS-induced hypertrophic signaling pathway and suppression of TGF- $\beta$ expression and TGF- $\beta$-induced collagen synthesis. Moreover, in cultured cardiomyocytes and myofibroblasts, recombinant kallistatin inhibited ROS formation through NO production. These combined findings indicate that kallistatin acts as an antioxidant in cardiac protection through NO formation.

\section{MATERIALS AND METHODS}

\section{Surgery in Rats and Adenoviral Vector Treatment}

MI was induced in male Wistar rats (250-300 g; Harlan, Indianapolis, IN, USA) by permanent ligation of the left coronary artery (LCA) as previously described. ${ }^{13}$ The investigation conformed to the Guide for the Care and Use of Laboratory Animals published by the US National Institutes of Health. Adenoviral vector harboring the human kallistatin cDNA under the control of the cytomegalovirus enhancer/ promoter (Ad.HKS) or adenoviral vector alone (Ad.Null) $\left(1 \times 10^{10}\right.$ plaque-forming units) was injected intramyocardially at six different sites along the border of infarcted area and noninfarcted area at $20 \mathrm{~min}$ after ligation. The survival rates in the sham, MI/Ad.Null and MI/Ad.HKS groups were 100, 70 and $73 \%$, respectively. At 4 weeks after MI surgery, survival rats ( $n=6$ in each group) were killed and the myocardium from the remote noninfarcted area in LV was immediately removed for analysis.

\section{Cardiac Hemodynamic Measurements}

At 4 weeks after MI, rats were anesthetized for hemodynamic measurements as previously described. ${ }^{12}$ A 2.5-French catheter (Millar Instrument, Houston, TX, USA) was introduced into the $\mathrm{LV}$ for measuring mean arterial pressure (MAP), left ventricular end diastolic pressure (LVEDP), maximum first derivative of pressure $(\mathrm{d} P / \mathrm{d} t$ max.) and minimum first derivative of pressure $(\mathrm{d} P / \mathrm{d} t \min$.). The data were recorded and analyzed by a model $7 \mathrm{E}$ polygraph (BIOPAC System Inc., Goleta, CA, USA).

\section{Histology and Immunohistochemistry}

Paraffin sections $(4 \mu \mathrm{m})$ were transversely obtained from the center of infarcted LV. Masson's trichrome staining was performed to distinguish areas of connective tissues and the extent of infarcted area. Cardiomyocyte size was determined by Gordon and Sweet's silver staining. A total of 40 cardiomyocytes in each noninfarcted LV were randomly chosen and traced with NIH Image software. Myocardial fibrosis was identified by sirius red staining and the percentage of collagen density in noninfarcted LV was analyzed by Adobe
PhotoShop software. Localization of recombinant human kallistatin in hearts was identified with a specific monoclonal antibody to human kallistatin as previously described. ${ }^{12}$ For immunostaining, slides were incubated with primary antibodies against collagen I (Chemicon, Temecula, CA, USA; 1:200) and collagen III (Santa Cruz Biotechnology, Santa Cruz, CA, USA; $1: 200)$ at $4^{\circ} \mathrm{C}$ overnight.

\section{Reverse Transcription-Polymerase Chain Reaction and Semi-Quantitative Real-Time PCR}

Reverse transcription-polymerase chain reaction (RT-PCR) for human kallistatin was performed using $5^{\prime}$ primers (CCACAACCTGAAGTTCCTTG) and 3' primers (GCCAGA AATGGAGAACTTGG). Real-time PCR was carried out using the gene expression assay of Rn005616661_ml for atrial natriuretic peptide (ANP), Rn00577357_ml for p22-phox, Rn01475962_ml for TGF- $\beta 1$ and Rn00561717_ml for plasminogen activator inhibitor-1 (PAI-1) (Applied Biosystems, Foster city, CA, USA). Gene expression was normalized by the housekeeping gene GAPDH (Mm99999915_gl).

\section{Measurements of Nitrate/Nitrite Levels, Superoxide Formation and NADH Oxidase Activity}

Nitrate/nitrite $\left(\mathrm{NO}_{x}\right)$ levels, an index of $\mathrm{NO}$ formation, in cardiac extracts were measured by a fluorometric assay as previously described. ${ }^{14}$ At 4 weeks after MI, superoxide levels in noninfarcted myocardium were measured by a spectrophotometric assay based on rapid reduction of ferricytochrome $c$ to ferrocytochrome $c{ }^{12} \mathrm{NADH}$ oxidase activities were measured by lucigenin-enhanced chemiluminescent detection using a luminometer (Turner Biosystems, Sunnyvale, CA, USA). ${ }^{12}$

\section{Primary Culture of Cardiomyocytes and Myofibroblasts}

Neonatal myofibroblasts were isolated from 1- to 3-day-old Sprague-Dawley rats by four rounds of digestion using collagenase II (Worthington, Lakewood, NJ, USA) and pancreatin (Sigma, St Louis, MO, USA). ${ }^{6}$ Cardiomyocytes were collected after $1 \mathrm{~h}$ of differential plating for myofibroblasts. Myofibroblasts were identified by positive immunostaining with $\alpha$-smooth muscle actin. Cardiomyocytes at 1-2 passages or myofibroblasts at 2-3 passages were used for in vitro studies. Recombinant human kallistatin was purified and characterized as previously described for use in the cell culture studies. ${ }^{15}$

\section{Intracellular Superoxide Determination and Dichlorofluoroscein Fluorescence Measurement}

Intracellular superoxide levels were determined by pretreating confluent cells with human kallistatin $(0.5 \mu \mathrm{mol} / \mathrm{l})$ with or without $N \omega$-nitro-L-arginine methyl ester (L-NAME, $200 \mu \mathrm{mol} / \mathrm{l}$ ) for $45 \mathrm{~min}$, followed by exposure to 0 or $200 \mu \mathrm{M}$ $\mathrm{H}_{2} \mathrm{O}_{2}$ for 40 min. ${ }^{16}$ After removing cultured medium, cells were incubated with dihydroethidium (HE, a probe for detecting intracellular superoxide, $10 \mu \mathrm{mol} / \mathrm{l}$ ) for $40 \mathrm{~min}$ and 
then photographed. For DCF fluorescence measurement, cells were washed with phenol red-free DMEM and pretreated with DCF diacetate $(20 \mu \mathrm{mol} / \mathrm{l})$ for $45 \mathrm{~min} .{ }^{17}$ After removing the probe, cells were incubated with $\mathrm{H}_{2} \mathrm{O}_{2}$ $(200 \mu \mathrm{mol} / \mathrm{l})$ in the presence or absence of kallistatin $(0.5 \mu \mathrm{mol} / \mathrm{l})$ or L-NAME $(200 \mu \mathrm{mol} / \mathrm{l})$. DCF fluorescence was recorded ( $485 \mathrm{~nm}$ excitation, $538 \mathrm{~nm}$ emission) for a period of $60 \mathrm{~min}$.

\section{Western Blotting}

Cytosolic fractions of noninfarcted myocardium were subjected to western blot for the detection of TGF- $\beta 1$ (Sigma; 1:1000). Cultured cardiomyocytes were serum-deprived, pretreated with or without kallistatin $(0.5 \mu \mathrm{mol} / \mathrm{l})$ for $45 \mathrm{~min}$, and then challenged with $\mathrm{H}_{2} \mathrm{O}_{2}(200 \mu \mathrm{mol} / \mathrm{l})$ in the presence or absence of kallistatin $(0.5 \mu \mathrm{mol} / \mathrm{l})$ for $15 \mathrm{~min}$, using cardiomyocytes cultured in serum-free medium as control. Cultured cardiomyocyte extracts underwent western blot analysis for the detection of phospho-Akt, total Akt, phosphoERK and total ERK (Cell Signaling Technology, Danvers, MA, USA; 1:1000). Conditioned medium from cultured myofibroblasts was collected after $24 \mathrm{~h}$ treatment with TGF- $\beta 1$ $(10 \mathrm{ng} / \mathrm{ml})$ with or without kallistatin $(0.5 \mu \mathrm{mol} / \mathrm{l})$ for the detection of collagen I by western blot (Santa Cruz; 1:400). Protein concentration was determined by Bio-Rad DC Protein Assay kit (Bio-Rad Laboratories, Hercules, CA, USA).

\section{Cell Survival Analysis}

Celltiter 96 AQueous One Solution Cell Proliferation Assay Kit (Promega, Madison, WI, USA) was used to measure viable cell levels according to manufacturer's instructions. After pretreatment with kallistatin $(1.0 \mu \mathrm{mol} / \mathrm{l})$ for $45 \mathrm{~min}$, cells were stimulated with $\mathrm{H}_{2} \mathrm{O}_{2}(750 \mu \mathrm{mol} / \mathrm{l})$ with or without kallistatin $(1.0 \mu \mathrm{mol} / \mathrm{l})$ for $24 \mathrm{~h}$. Absorbance readings were measured with a photometric reader after $2 \mathrm{~h}$ incubation with a solution of tetrazolium compound (MTS).

\section{Transient Transfection and NF- $\kappa$ B Luciferase Activity Assay}

A nucleofector kit for primary cardiomyocytes (Amaxa Biosystems, Gaithersburg, MD, USA) was used for transient transfection according to the manual bulletin. One day after transfection, cells were serum-deprived for $24 \mathrm{~h}$ and then were incubated with $\mathrm{H}_{2} \mathrm{O}_{2}(200 \mu \mathrm{mol} / \mathrm{l})$ with or without kallistatin $(0.5 \mu \mathrm{mol} / \mathrm{l})$ for $24 \mathrm{~h}$. Cells were lysed in lysis buffer, and NF- $\kappa \mathrm{B}$ firefly luciferase activity was measured with luciferase assay reagent (Promega). The data from three independent transfections were normalized with protein concentrations.

\section{Collagenase-Sensitive $\left[{ }^{3} \mathrm{H}\right]$-Proline Incorporation}

Collagenase-sensitive $\left[{ }^{3} \mathrm{H}\right]$-proline incorporation was measured for collagen synthesis in cultured myofibroblasts. ${ }^{18}$ Growth-arrested myofibroblasts were pulsed by adding $1 \mu \mathrm{Ci} / \mathrm{ml}\left[{ }^{3} \mathrm{H}\right]$-proline (PerkinElmer Life Sciences, Waltham,
MA, USA) and $10 \mathrm{ng} / \mathrm{ml}$ TGF- $\beta 1$ with or without kallistatin $(0.5 \mu \mathrm{mol} / \mathrm{l})$ for $48 \mathrm{~h}$. Incorporated $\left[{ }^{3} \mathrm{H}\right]$-proline after $2 \mathrm{mg} / \mathrm{ml}$ collgenase II (Worthington) digestion was counted by a liquid scintillation counter.

\section{Statistical Analysis}

Data were expressed as mean \pm s.e.m. and were compared among experimental groups with the use of one-way ANOVA followed by Newman-Keuls multiple comparison test. A difference of $P<0.05$ was considered statistically significant.

\section{RESULTS}

\section{Expression of Recombinant Human Kallistatin in Rat Hearts}

Four weeks after local injection of adenovirus containing the human kallistatin gene in rat hearts, expression and localization of recombinant human kallistatin protein and mRNA were identified in both infarcted and noninfarcted areas by immunohistochemistry (Figure 1a) and by RT-PCR (Figure 1b). Moreover, using a human kallistatin specific ELISA, ${ }^{12}$ immunoreactive human kallistatin levels in noninfarcted left ventricular extracts were $0.54 \pm 0.23 \mathrm{ng} / \mathrm{mg}$ protein $(n=4)$ and $0.10 \pm 0.01 \mathrm{ng} / \mathrm{mg}$ protein $(n=4)$ at 1 and 4 weeks, respectively, after kallistatin gene delivery. Neither human kallistatin mRNA nor protein was detectable in the corresponding tissues of rats injected with control virus. Human kallistatin was undetectable in the circulation after local gene delivery, and serum protein levels of rat kallistatin remained unchanged in all groups, as determined by specific human and rat kallistatins (data not shown).

\section{Kallistatin Gene Delivery Improves Hemodynamic Parameters}

The effects of kallistatin on cardiac function at 4 weeks after MI are shown in Table 1. Heart weight (HW) to body weight (BW) ratio was increased by $12.3 \%$ in MI/Ad.Null group, and increased by only $6.3 \%$ after Ad.HKS treatment, compared with the sham group. However, there was no statistical difference in HW/BW among the groups. MAP was decreased in the MI control group compared with the sham group, and kallistatin treatment significantly restored MAP levels. Rats injected with Ad.Null gene showed decreased cardiac contractile and relaxing function, as indicated by increased LVEDP and decreased $\mathrm{d} P / \mathrm{d} t(\mathrm{~d} P / \mathrm{d} t$ max and $\mathrm{d} P / \mathrm{d} t \min )$. Kallistatin gene transfer significantly improved cardiac performance.

\section{Kallistatin Gene Delivery Reduces Infarct Size and Restores Left Ventricular Wall Thickness}

Representative Masson's trichrome staining showed that kallistatin treatment reduced infarcted size after MI (Figure 1c). Quantitative analysis indicated that kallistatin gene transfer significantly reduced the ratio of infarcted area to area at risk from $37.4 \pm 2.6$ to $28.6 \pm 3.0 \%$ at 4 weeks after MI $(P<0.05, n=6)$ (Figure 1d). Moreover, LV wall thickness was 
a

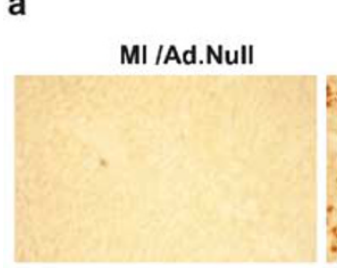

b
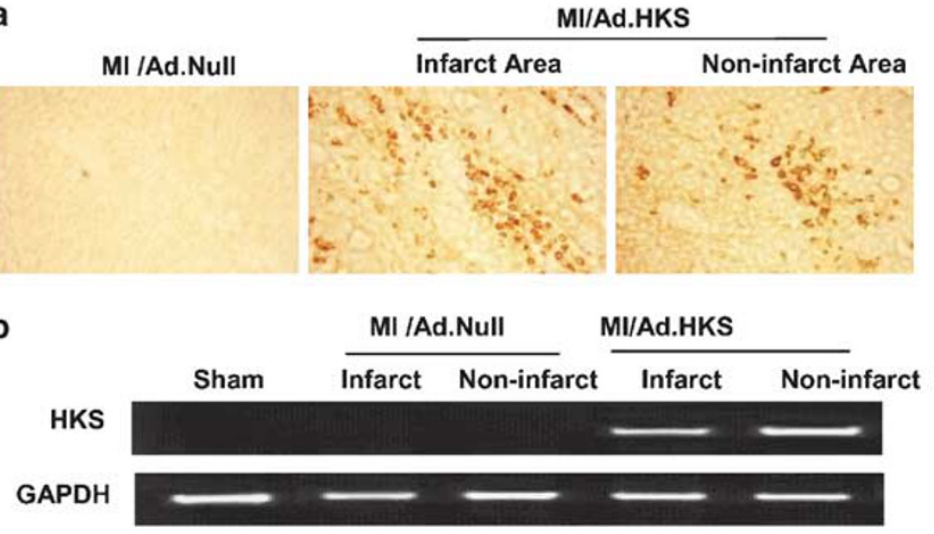

C

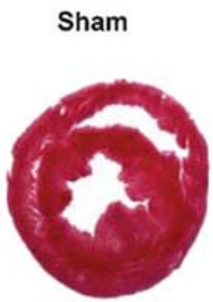

MI/Ad.Null

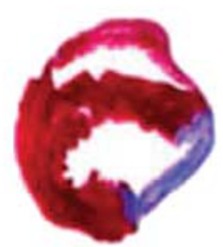

MI/Ad.HKS

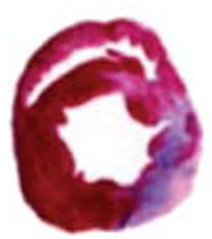

d

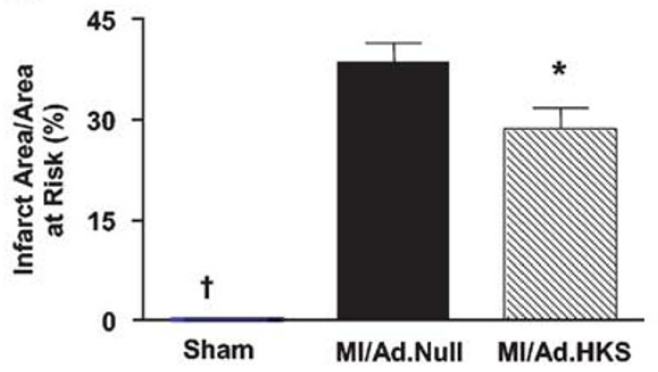

e

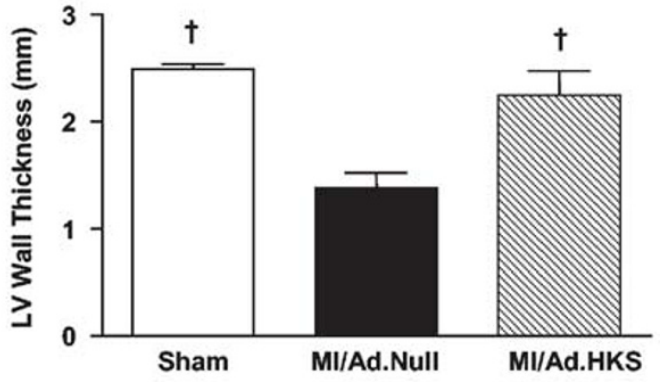

Figure 1 Expression and localization of kallistatin and effects of kallistatin on infarct size and LV wall thickness at 4 weeks after MI. (a) Identification of human kallistatin protein levels immunohistochemical staining (original magnification is $\times 200$ ) in both infarct area and noninfarct area myocardium. (b) mRNA expression of human kallistatin by RT-PCR. (c) Representative photographs of Masson's trichrome staining to depict infarct size (original magnification is $\times 50$ ). (d) Analysis of infarcted area is expressed as a ratio of infarcted area to area at risk in LV. (e) LV wall thickness quantification results; ${ }^{\star} P<0.05,{ }^{\dagger} P<0.01$ vs MI/Ad.Null. Data are expressed as mean \pm s.e.m., $n=6$.

Table 1 Effects of kallistatin gene delivery on cardiac function at 4 weeks after MI

\begin{tabular}{lccc}
\hline & Sham & Ml/Ad.Null & MI/Ad.HKS \\
\hline HW/BW $(\mathrm{g} / \mathrm{kg})$ & $3.50 \pm 0.15$ & $3.93 \pm 0.20$ & $3.72 \pm 0.05$ \\
MAP $(\mathrm{mm} \mathrm{Hg})$ & $109.50 \pm 3.12^{\dagger}$ & $58.25 \pm 3.12$ & $70.00 \pm 3.30^{*}$ \\
LVEDP $(\mathrm{mm} \mathrm{Hg})$ & $1.54 \pm 0.32^{\dagger}$ & $15.19 \pm 1.34$ & $9.47 \pm 1.21^{\dagger}$ \\
$\mathrm{dP} / \mathrm{d} t$ max. $(\mathrm{mm} \mathrm{Hg} / \mathrm{s})$ & $3994.17 \pm 87.47^{\dagger}$ & $2032.13 \pm 96.75$ & $2560.33 \pm 104.63^{\dagger}$ \\
DP/dt min. $(\mathrm{mm} \mathrm{Hg} / \mathrm{s})$ & $3530.33 \pm 90.40^{\dagger}$ & $1465.50 \pm 105.74$ & $1926.33 \pm 139.40^{\dagger}$
\end{tabular}

BW, body weight; $\mathrm{d} P / \mathrm{d} t$ max., maximum first derivative of pressure; $\mathrm{d} P / \mathrm{d} t$ min., minimum first derivative of pressure; HW, heart weight; LVEDP, left ventricular end diastolic pressure; MAP, mean arterial pressure.

Values are presented as mean \pm s.e.m.

${ }^{*} P<0.05,{ }^{\dagger} P<0.01$ vs Ml/Ad.Null $(n=6)$.

significantly reduced by permanent MI compared with sham, but was restored by kallistatin gene delivery (Figure 1e).

\section{Kallistatin Attenuates Cardiac Hypertrophy}

Representative Gordon and Sweet's silver staining showed that kallistatin reduced enlarged cardiomyocyte size in noninfarcted area at 4 weeks after MI (Figure 2a). Quantitative analysis showed that kallistatin gene delivery significantly reduced cardiomyocyte size compared with the MI/Ad.Null group (Figure 2b). The inhibitory effect of kallistatin on hypertrophy was associated with a significant reduction of ANP mRNA levels, a marker of cardiac hypertrophy (Figure 2c).

\section{Kallistatin Attenuates Collagen Deposition and Expression of Profibrotic Factors}

Examination and quantitative analysis of heart sections by sirius red staining indicated that kallistatin reduced MI-induced collagen deposition and collagen fraction volume (Figure 3a and b). Similarly, immunostaining showed that kallistatin reduced collagen types I and III levels (Figure 3a). Reduction of collagens I and III accumulation by kallistatin was further confirmed by measurements of their mRNA levels (Figure $3 \mathrm{c}$ and d). Western blot and quantitative analysis showed that kallistatin treatment prevented elevation of TGF- $\beta 1$ levels induced by MI (Figure 3e). Kallistatin also reduced TGF- $\beta 1$ and PAI- 1 mRNA levels in noninfarcted myocardium (Figure $3 \mathrm{f}$ and $\mathrm{g}$ ).

\section{Kallistatin Increases NO Formation and Reduces Oxidative Stress after MI}

At 4 weeks after MI, kallistatin gene delivery significantly increased cardiac $\mathrm{NO}_{x}$ content compared with sham and MI groups (Figure 4a). Kallistatin gene delivery significantly inhibited superoxide formation and NADH oxidase activity compared with the MI/Ad.Null group in the noninfarcted myocardium (Figure $4 \mathrm{~b}$ and $\mathrm{c}$ ). Furthermore, inhibition of 
a

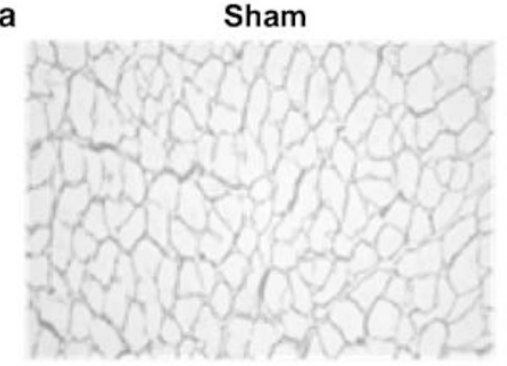

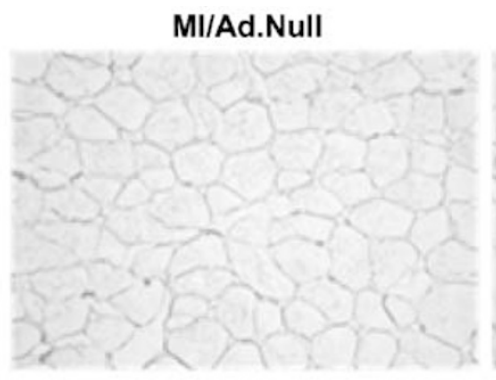
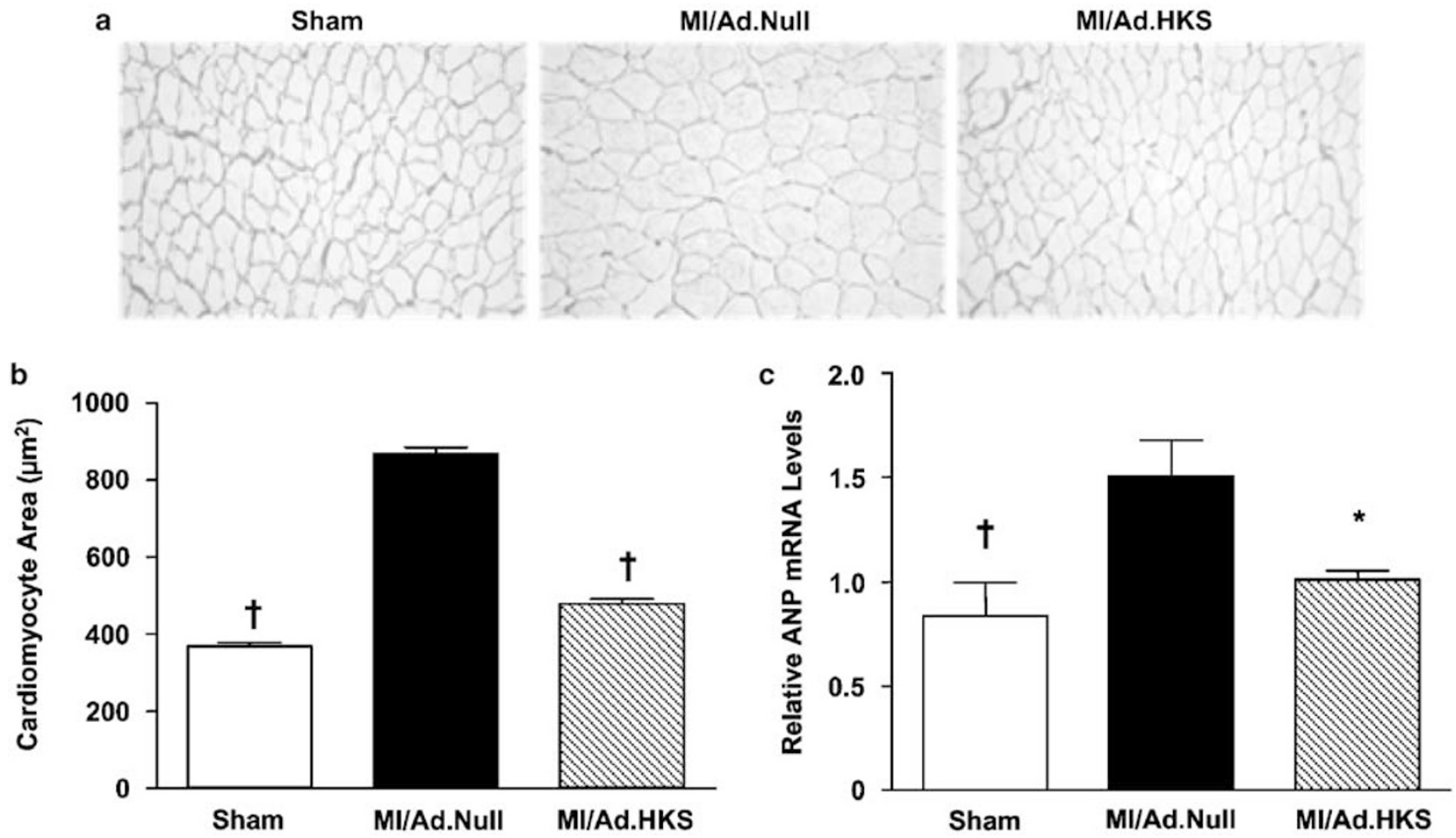

Figure 2 Kallistatin prevents cardiac hypertrophy at 4 weeks after MI. (a) Cardiomyocyte size was visualized by silver staining in noninfarcted myocardium (original magnification is $\times 400$ ). (b) Quantification analysis of cardiomyocyte size from silver staining. (c) ANP mRNA expression in noninfarcted myocardium by real-time PCR; ${ }^{*} P<0.05,{ }^{\dagger} P<0.01$ vs Ml/Ad.Null. Data are expressed as mean \pm s.e.m., $n=6$.

NADH oxidase activity by kallistatin was accompanied by a reduction in p22-phox mRNA levels (Figure 4d).

\section{Effects of Recombinant Kallistatin on ROS Formation and ROS-Induced Prohypertrophic Signaling Pathway in Cultured Cardiomyocytes}

In primary cardiomyocytes, recombinant kallistatin inhibited $\mathrm{H}_{2} \mathrm{O}_{2}$-induced intracellular superoxide formation as determined by HE fluorescence intensity, and the inhibitory effect of kallistatin on in situ ROS formation was abrogated by L-NAME, indicating a NO-mediated event (Figure 5a). $\mathrm{H}_{2} \mathrm{O}_{2}$-induced DCF fluorescence in a time-dependent manner and kallistatin inhibited the increases (Figure 5b). Again, kallistatin's effect on $\mathrm{H}_{2} \mathrm{O}_{2}$-induced ROS formation was blocked by L-NAME. Kallistatin significantly promoted cell survival (Figure $5 \mathrm{c}$ ) and inhibited $\mathrm{H}_{2} \mathrm{O}_{2}$-stimulated Akt and ERK phosphorylation (Figure $5 \mathrm{~d}$ ) and NF- $\kappa \mathrm{B}$ activation (Figure 5e) in cultured cardiomyocytes.

\section{Effect of Recombinant Kallistatin on ROS Formation, and TGF- $\beta$-Induced Collagen Synthesis in Cultured Myofibroblasts}

In myofibroblasts, kallistatin inhibited $\mathrm{H}_{2} \mathrm{O}_{2}$-induced intracellular oxidant production and the effect was blocked by L-NAME as determined by both HE and DCF fluorescence measurements (Figure 6a and b). Kallistatin significantly inhibited TGF- $\beta 1$-induced $\left[{ }^{3} \mathrm{H}\right]$-proline incorporation
(Figure 6c) in cultured myofibroblasts. To confirm that the observed effect was specific to the synthesis of collagen I, immunoblotting was used to analyze non-cross-linked collagen secreted into the cultured medium. Kallistatin inhibited TGF- $\beta$ 1-induced collagen I secretion in the medium of cultured myofibroblasts (Figure 6d).

\section{DISCUSSION}

This study identified a novel role of kallistatin in preventing cardiac injury and remodeling by inhibiting oxidative stress and ROS-related hypertrophy and fibrosis in an animal model of postinfarcted and CHF. Our lab has previously shown that kallistatin plays a protective role in heart during acute ischemia by preventing apoptosis and inflammation in infracted myocardium. ${ }^{12}$ This study adds to the previous work by showing the longer-term benefits of kallistatin in MI. We demonstrate here that administration of kallistatin prevents cardiac fibrosis and hypertrophy, leading to a reduction in cardiac remodeling. This study show that ROS levels are diminished after kallistatin gene delivery suggesting kallistatin may act as an antioxidant to prevent cardiac remodeling and oxidative cardiac damage. Moreover, kallistatin was found to reduce ROS formation and inhibit ROS-related prohypertrophic and profibrotic signaling pathway. Our previous work focused on the acute effects of kallistatin with myocardial ischemia and reperfusion, whereas this study investigated chronic effects of MI and the ability of kallistatin 


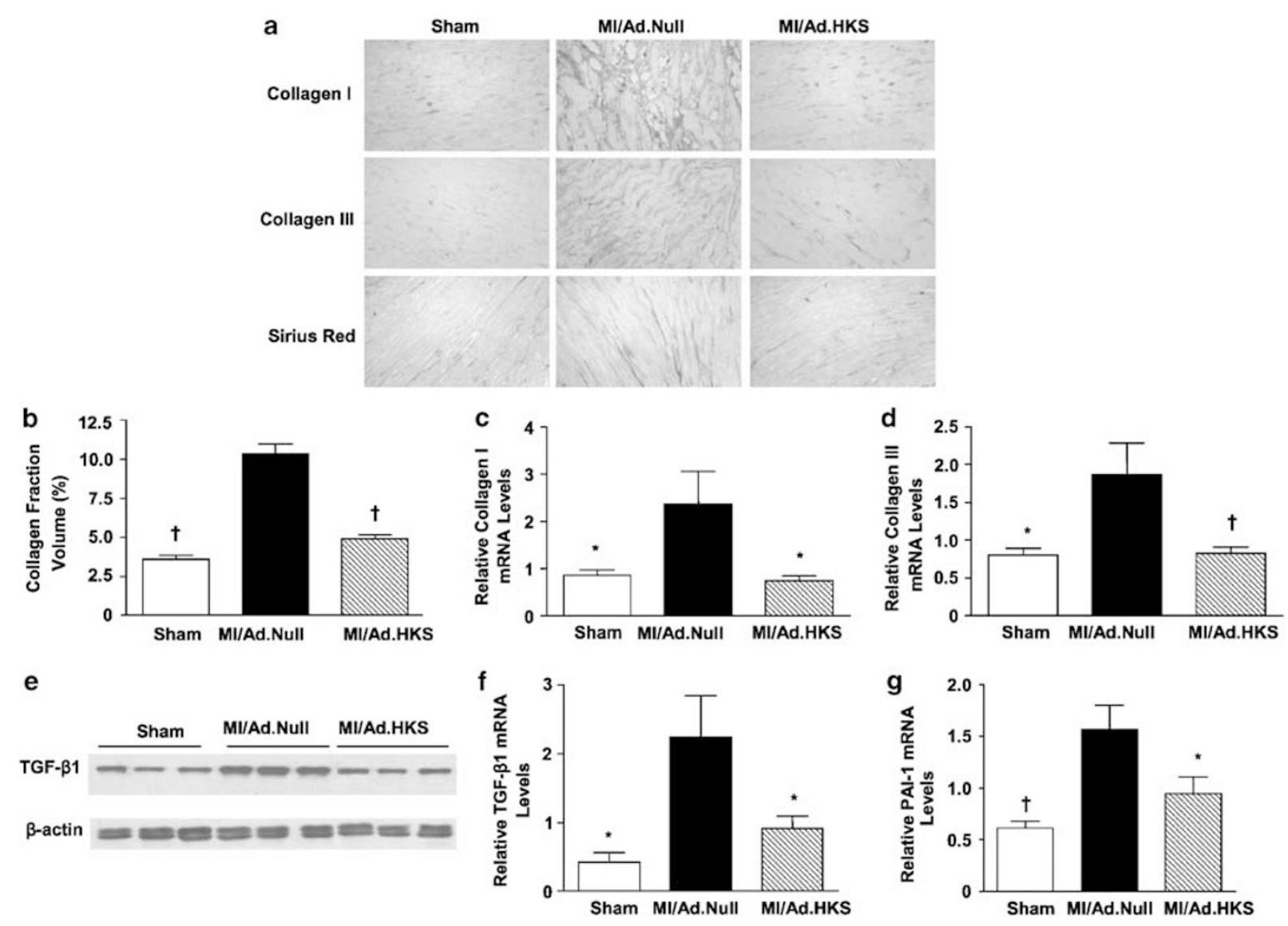

Figure 3 Kallistatin reduces collagen deposition and TGF- $\beta 1$ and PAI-1 levels in noninfarcted myocardium. (a) Representative sections of sirius red staining and immunohistochemistry of collagens I and III (original magnification is $\times 400$ ). (b) Collagen fraction volume resulting from quantification of sirius red staining. (c) Collagen I and (d) collagen III mRNA expression by real-time PCR in noninfarcted myocardium. (e) Representative TGF- $\beta 1$ protein levels in noninfarcted myocardium by western blot. (f) TGF- $\beta 1$ and (g) PAI- 1 mRNA levels determined by real-time PCR in noninfarcted myocardium; ${ }^{*}<<0.05$, ${ }^{\dagger} P<0.01$ vs Ml/Ad.Null. Data are expressed as mean \pm s.e.m., $n=6$.
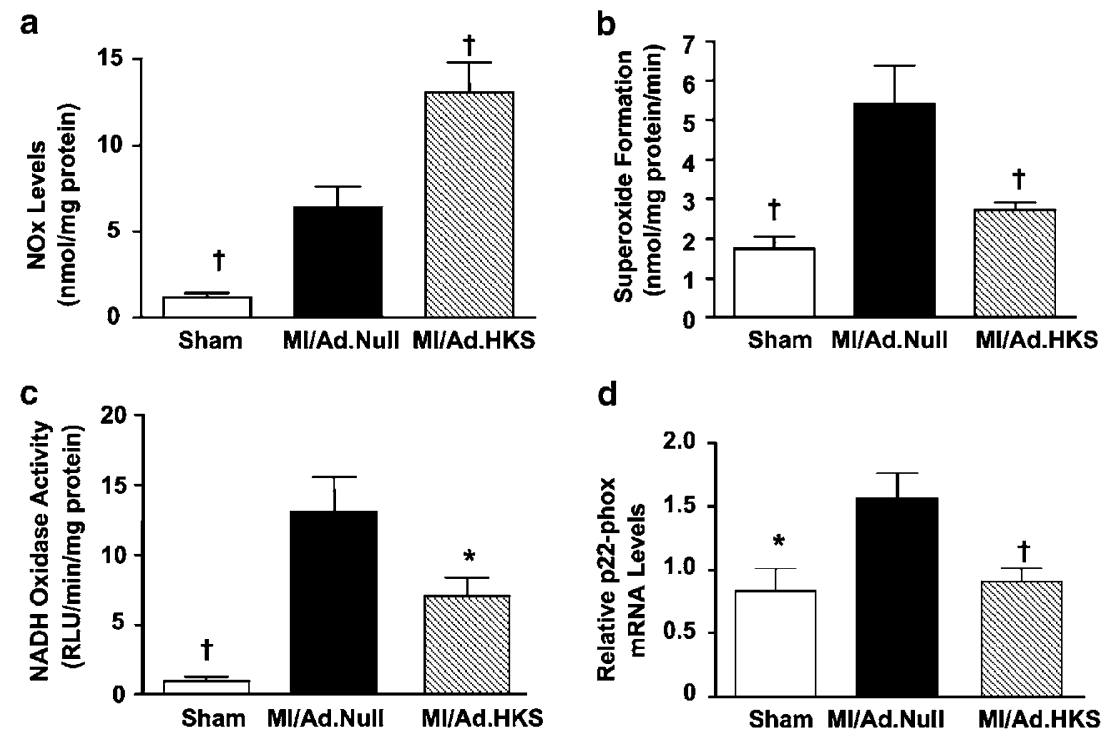

Figure 4 Kallistatin increases $\mathrm{NO}_{x}$ levels, and inhibits ROS formation and NADH oxidase activity. (a) NO levels, (b) superoxide anion formation, (c) LV NADH oxidase activity and (d) p22-phox mRNA expression in noninfarcted myocardium at 4 weeks after $\mathrm{Ml} ;{ }^{*} P<0.05,{ }^{\dagger} P<0.01$ vs $\mathrm{Ml} /$ Ad.Null. Data are expressed as mean \pm s.e.m., $n=6$. 
a

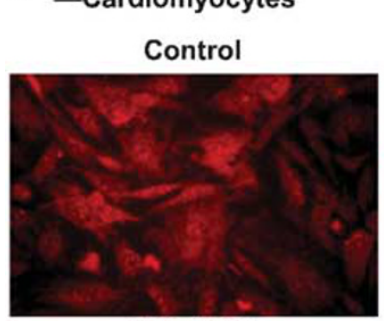

$\mathrm{H}_{2} \mathrm{O}_{2} / \mathrm{HKS}$
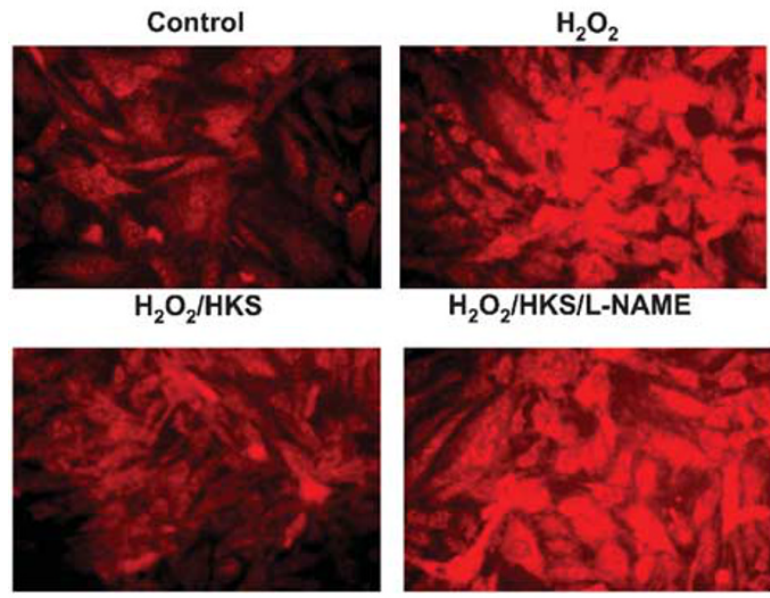

$\mathrm{H}_{2} \mathrm{O}_{2}$ /HKS/L-NAME

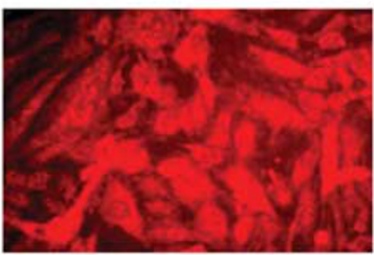

b

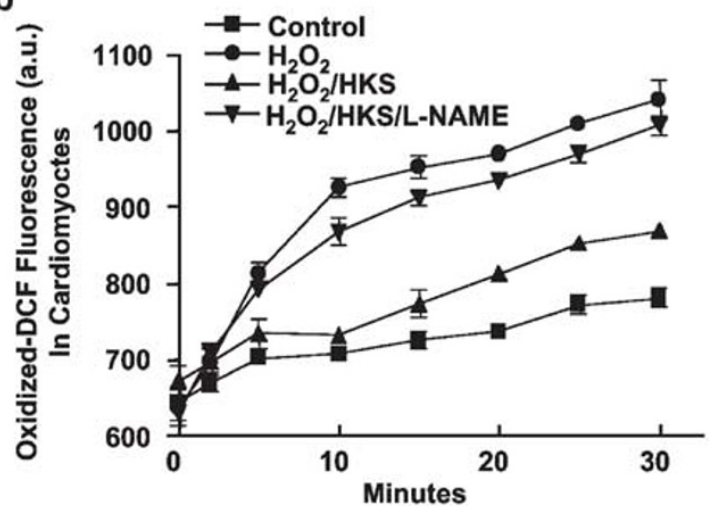

c

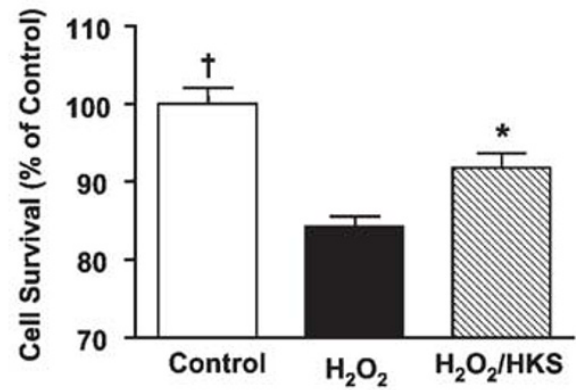

d

Phospho-Akt

Total-Akt

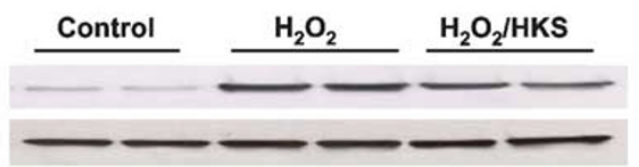

Phospho-ERK

Total-ERK

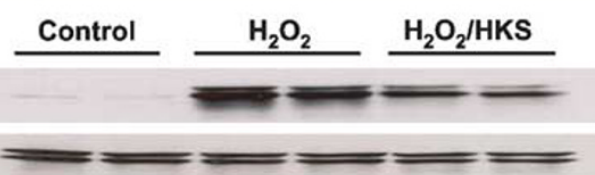

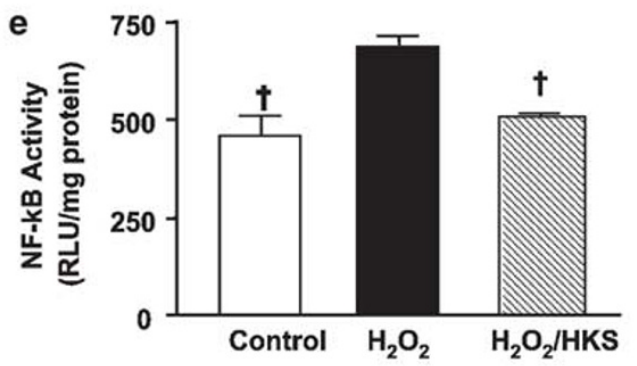

Figure 5 Kallistatin inhibits ROS formation and ROS-induced hypertrophic signaling in cardiomyocytes. Detection of intracellular ROS by (a) in situ HE fluorescence staining and (b) oxidized DCF fluorescence measurement (a.u., arbitrary units). (c) Cell survival determined by MTS assay. (d) Western blots of phosphorylated and total Akt and ERK. (e) NF- $\kappa$ B activation in cultured cardiomyocytes (RLU, relative light units); ${ }^{\star} P<0.05,{ }^{\dagger} P<0.01$ vs $\mathrm{H}_{2} \mathrm{O}_{2}$. Data from independent triplicate experiments are expressed as mean \pm s.e.m., $n=3$.

to modulate them. We believe that kallistatin affords cardiac protection both at the early and later stages of cardiac remodeling induced by myocardial ischemia.

It is well known that oxidative stress causes cardiomyocyte death, ventricular dysfunction and remodeling after MI, thus contributing to high mortality in CHF patients. Our results showed that kallistatin increased $\mathrm{NO}_{x}$ levels and inhibited $\mathrm{NADH}$ oxidase activity and superoxide formation through downregulation of p22-phox expression in failing hearts at 4 weeks after MI. These results indicate a clue that, through NO formation, kallistatin prevented hearts from undergoing cardiac remodeling by inhibiting oxidative stress and ROSinduced cardiomyocyte apoptosis, cardiac hypertrophy and fibrosis.

Ischemia-generated ROS-induced cardiomyocyte apoptosis and necrotic cell death. ${ }^{19}$ Our current study showed that kallistatin improved the survival of cultured cardiomyocytes subjected to high levels of ROS. At 4 weeks after MI, a mature scar had already formed in the area of infarction, being mainly consisting of acellular and fibrillar collagen. Thus, at this point, apoptotic cells and signals were undetectable in the infarcted heart. Reduction of infarcted size and restoration of LV wall thickness also provide direct evidence for increased cardiomyocyte survival after chronic MI. This finding is consistent with our previous study showing that kallistatin gene transfer inhibited cardiomyocyte apoptosis and inflammation in infarcted myocardium after acute ischemia/reperfusion injury. ${ }^{12}$ Thus, considering that reversible cardiomyocyte injury during the acute phase is important for the subsequent ventricular remodeling in postinfarcted heart failure, ${ }^{20}$ the protective effects of kallistatin observed in the chronic phase may be partially attributed to its ability to improve cardiomyocyte survival under oxidative stress damage or attenuate cardiomyocyte apoptosis and inflammation in the early phase after MI.

It is well known that the development of hypertrophy is tightly coupled with ROS, which trigger redox-related hypertrophic signaling pathways. Among them, the PI3-kinase/ 
a -myofibroblasts
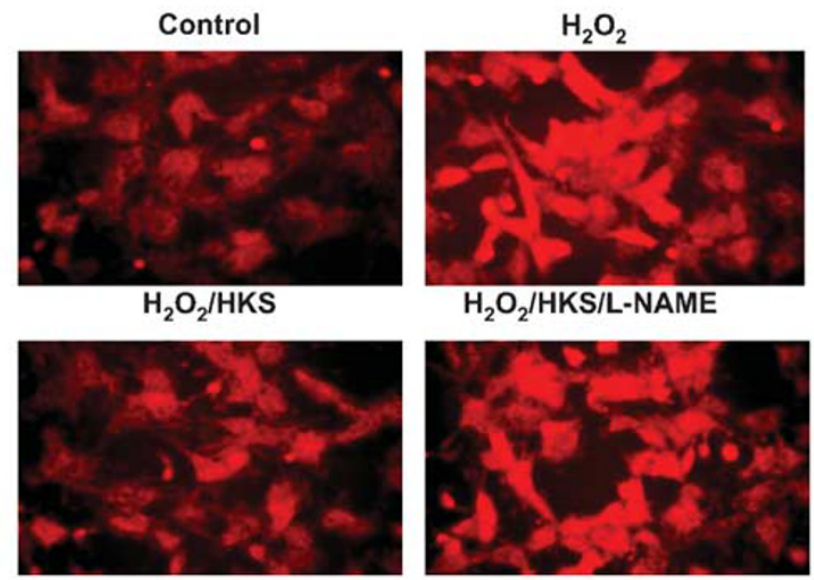

$\mathrm{H}_{2} \mathrm{O}_{2}$ /HKS/L-NAME

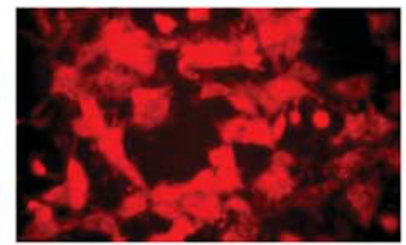

b

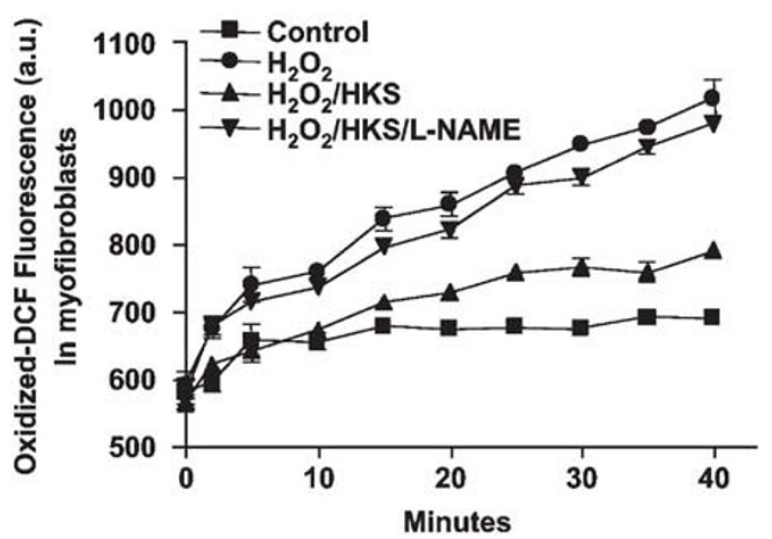

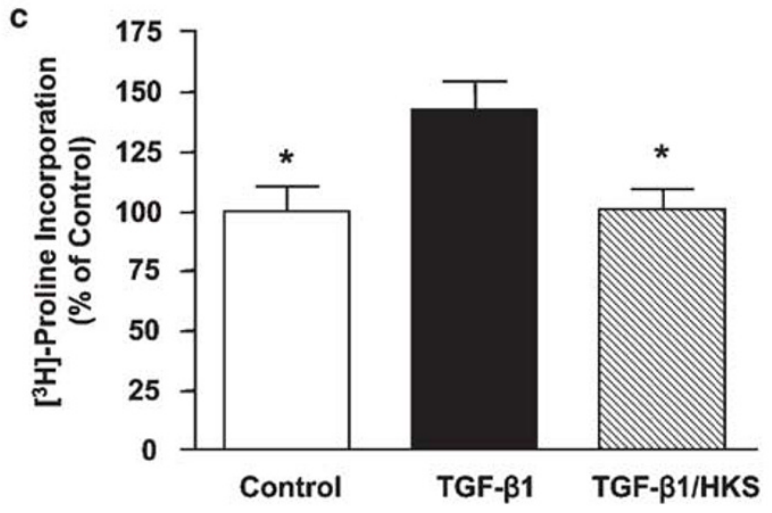

d

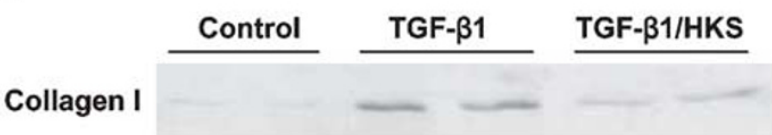

Figure 6 Kallistatin abrogates ROS levels and inhibits TGF- $\beta$-induced collagen synthesis and secretion in cultured myofibroblasts. Determination of intracellular ROS by (a) in situ HE fluorescence staining and (b) oxidized DCF fluorescence measurement. (c) TGF- $\beta 1$-induced collagen synthesis determined by collagenase-sensitive $\left[{ }^{3} \mathrm{H}\right]$-proline incorporation. (d) TGF- $\beta 1$-induced collagen secretion in cultured medium from myofibroblasts; ${ }^{*} P<0.05,{ }^{\dagger} P<0.01$ vs TGF- $\beta 1$. Data from independent triplicate experiments are expressed as mean \pm s.e.m., $n=3$.

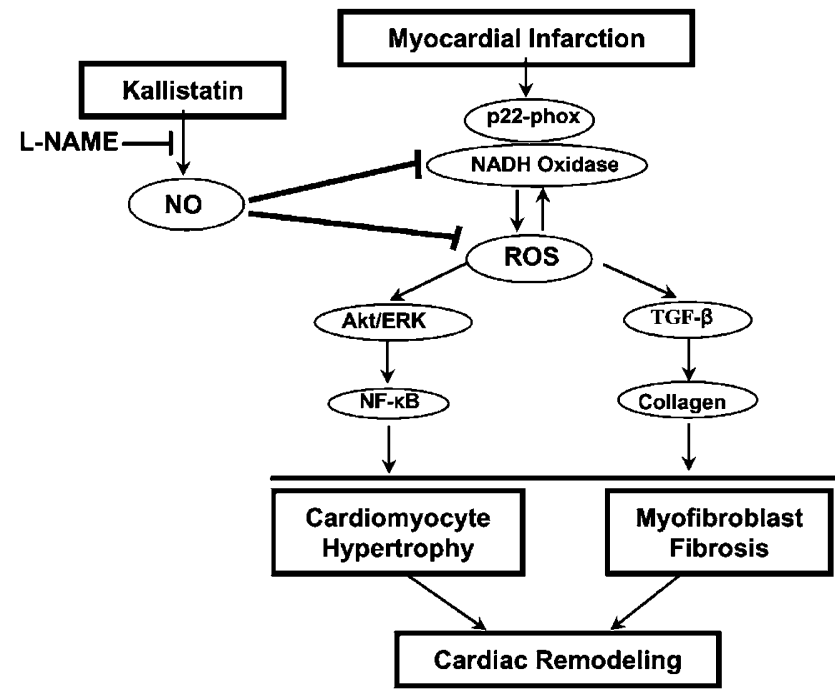

Figure 7 Schematic diagram of kallistatin through inhibition of oxidative stress and protection against cardiac remodeling after ischemic infarction.
Akt pathway is required for transition from compensated to decompensated hypertrophy, as transgenic expression of Akt in the heart was shown to lead to an increase in cardiomyocyte size. ${ }^{4,21}$ In addition, ANP, a cardiac hypertrophy marker, is activated in an Akt-dependent manner. ${ }^{22}$ However, one study reported that short-term activation of Akt-induced cardiac hypertrophy without ANP gene expression. ${ }^{23}$ Moreover, ERK is considered to be activated by mechanical stress, which induces a G-protein-coupled hypertrophic stimulation in heart failure. ${ }^{24}$ The transcription factor NF- $\kappa$ B has also been observed to be activated by ROS and is responsible for the induction of hypertrophy in cardiomyocytes. ${ }^{25}$ In this study, kallistatin therapy minimized postinfarcted LV hypertrophy evidenced by diminished HW/ BW, cardiomyocyte size, ANP expression, as well as inhibition of Akt/ERK/NF- $\kappa$ B activation induced by $\mathrm{H}_{2} \mathrm{O}_{2}$. Our results indicate that, under oxidative stress circumstances induced by MI, the antihypertrophic effect of kallistatin is, at least partially, mediated by the inhibition of ROS-related prohypertrophic signaling pathway in cardiomyocytes (Figure 7). 
Fibrosis is one of the most refractory clinical problems at the late stage of cardiac remodeling. ${ }^{26}$ At this stage, TGF- $\beta 1$ activates fibrogenic pathways by inducing extracellular matrix deposition and contributes to the pathogenesis of left ventricular remodeling by promoting fibrosis of the noninfarcted myocardium. ${ }^{18,27}$ TGF- $\beta$ can also prevent the degradation of collagen via increased PAI-1 expression. ${ }^{17,28}$ It has been reported that ROS modulate fibroblast proliferation and their transition into matrix-producing myofibroblasts in the heart. ${ }^{5,29}$ Furthermore, ROS induce expression of TGF- $\beta 1$ and type I collagen, and lead to activation of TGF- $\beta$ from its latent form. ${ }^{6,70}$ Treatment with the antioxidant taurine was shown to attenuate hepatic fibrosis by inhibiting oxidative stress and TGF- $\beta$ gene expression. ${ }^{31}$ In this study, kallistatin decreased the levels of the profibrotic molecules such as TGF- $\beta 1$ and PAI- 1 in the ischemic myocardium, and directly inhibited TGF- $\beta 1$-induced collagen I synthesis and secretion in cultured myofibroblasts, thus preventing the occurrence and expansion of cardiac fibrosis (Figure 7).

After ischemic injury, endogenous ROS formation increases due to an imbalance of oxidant-generating and -catalyzing systems. Superoxide anion, the primary precursor in ROS metabolic pathways, decreases NO bioavailability and attenuates vasodilatation, thus increasing cardiac loading in $\mathrm{CHF}^{32} \mathrm{NO}$ is known to abolish mitochondrial oxidant damage and calcium overload, and reduce superoxide anion production via an efficient suppression on the activity of $\mathrm{NAD}(\mathrm{P}) \mathrm{H}$ oxidase and the assembly of $\mathrm{NAD}(\mathrm{P}) \mathrm{H}$ oxidase subunits. ${ }^{1,33}$ Maintenance of NO levels is critical for recovery of heart failure after ischemic injury. As part of a self-protective process, we found that CHF causes a modest increase in interstitial NO levels (consistent with a previous publication $^{34}$ ) and may be derived from activated NOS isoforms and/or a NOS-independent pathway. ${ }^{35,36}$ We have previously reported that kallistatin treatment increased NO levels through activating endothelial nitric oxide synthase (eNOS) in infarcted myocardium at 1 day after acute ischemia/reperfusion injury. ${ }^{12}$ The present study showed that at 4 weeks after permanent LCA ligation, kallistatin gene transfer also significantly increased $\mathrm{NO}$ levels and inhibited ROS formation in noninfarcted myocardium, compared with Ad.Null group. However, we found that kallikstatin treatment increased cardiac NO levels in conjunction with elevation of inducible NOS (iNOS) expression in noninfarcted myocardium. Similarly, kallistatin also stimulated iNOS expression in cultured cardiomyocytes and myofibroblasts (data not shown). Moreover, our study found that inhibition of NOS by L-NAME abolished the antioxidative effect of kallistatin on ROS formation in both cardiomyocytes and myofibroblasts. As long-term treatment with L-NAME could cause unwanted side effects such as hypertension in vivo, L-NAME was used only in vitro to determine the effects of NO. As reported, iNOS was initially perceived to generate $\mathrm{NO}$ in order to protect cells from apoptosis under chronic conditions, especially in proin- flammatory situations. ${ }^{37,38}$ Moreover, in iNOS knockout mice, the antoxidant resveratrol had no protective effects on attenuating cardiac injury, though it protected against cardiac damage in wild-type mice, supporting a protective role of iNOS-related $\mathrm{NO}$ as an antioxidant. ${ }^{39}$ NO formation through increased NOS activity should be an important protective mechanism during kallistatin treatment after MI injury.

Taken together, these findings indicate that kallistatin inhibits ROS injury through NO formation and thus protects against cardiomyocyte apoptosis, necrosis, hypertrophy and fibrosis by suppressing ROS-induced Akt/ERK and NF- $\kappa \mathrm{B}$ activation and ANP, TGF- $\beta 1$, PAI- 1 and collagen expression (Figure 7). Kallistatin should be recognized as an important regulator in protection of post-MI injury and cardiac remodeling.

\section{ACKNOWLEDGEMENTS}

This work was supported by National Institutes of Health grant HL-44083 and C06 RR015455 from the Extramural Research Facilities Program of the National Center for Research Resources.

1. Murdoch $C E$, Zhang $M$, Cave $A C$, et al. NADPH oxidase-dependent redox signaling in cardiac hypertrophy, remodeling and failure. Cardiovasc Res 2006;71:208-215.

2. Irani K. Oxidant signaling in vascular cell growth, death, and survival: a review of the roles of reactive oxygen species in smooth muscle and endothelial cell mitogenic and apoptotic signaling. Circ Res 2000;87:179-183.

3. Takimoto E, Kass AD. Role of oxidative stress in cardiac hypertrophy and remodeling. Hypertension 2007;49:241-248.

4. Padmasekar $M$, Nandigama $R$, Wartenberg $M$, et al. Cardiovasc Res 2007;75:118-128.

5. Murrell AC, Francis JO, Bromley L. Modulation of fibroblast proliferation by oxygen free radicals. Biochem J 1990;265:659-665.

6. Siwik DA, Pagano PJ, Colucci WS. Oxidative stress regulates collagen synthesis and matrix metalloproteinase activity in cardiac fibroblasts. Am J Physiol Cell Physiol 2001;280:C53-C60.

7. Wang $\mathrm{H}$, Kochevar IE. Involvement of UVB-induced reactive oxygen species in TGF- $\beta$ biosynthesis and activation in keratinobytes. Free Radic Biol Med 2005;38:890-897.

8. Heitzer T, Schlinzig T, Krohn K, et al. Endothelial dysfunction, oxidative stress, and risk of cardiovascular events in patients with coronary artery diseases. Circulation 2001;104:2673-2678.

9. Chao J, Stallone JN, Liang YM, et al. Kallistatin is a potent new vasodilator. J Clin Invest 1997;100:11-17.

10. Miao RQ, Agata J, Chao L, et al. Kallistatin is a new inhibitor of angiogenesis and tumor growth. Blood 2002;100:3245-3252.

11. Wang CR, Chen SY, Wu CL, et al. Prophylactic adenovirus-mediated human kallistatin gene therapy suppresses rat arthritis by inhibiting angiogenesis and inflammation. Arthritis Rheum 2005;52:1319-1324.

12. Chao J, Yin $\mathrm{H}$, Yao YY, et al. Novel role of kallistatin in protection against myocardial ischemia-reperfusion injury by preventing apoptosis and inflammation. Hum Gene Ther 2006;17:1201-1213.

13. Pfeffer MA, Pfeffer JM, Steinberg C, et al. Survival after an experimental myocardial infarction: beneficial effects of long-term therapy with captopril. Circulation 1985;72:406-412.

14. Misko TP, Schilling RJ, Salvemini $D$, et al. A fluorometric assay for the measurement of nitrite in biological samples. Anal Biochem 1993;214:11-16.

15. Miao RQ, Chen V, Chao L, et al. Structural elements of kallistatin required for inhibition of angiogenesis. Am J Physiol Cell Physiol 2003;284:C1604-C1613.

16. Li WG, Miller FJ, Zhang $\mathrm{HJ}$, et al. $\mathrm{H}_{2} \mathrm{O}_{2}$-induced $\mathrm{O}_{2}^{-}$production by a non-phagocytic NAD(P)H oxidase causes oxidant injury. J Biol Chem 2001;276:29251-29256. 
17. Matsumoto $\mathrm{H}$, Ishikawa $\mathrm{K}$, Itabe $\mathrm{H}$, et al. Carbon monoxide and bilirubin from heme oxygenase- 1 suppresses reactive oxygen species generation and plasminogen activator inhibitor-1 induction. Mol Cell Biochem 2006;291:21-28.

18. Swaney JS, Roth DM, Olson ER, et al. Inhibition of cardiac myofibroblast formation and collagen synthesis by activation and overexpression of adenylyl cyclase. Proc Natl Acad Sci USA 2005;102:437-442.

19. Ide $T$, Tsutsui $H$, Hayashidani $S$, et al. Mitochondrial DNA damage and dysfunction associated with oxidative stress in failing hearts after myocardial infarction. Circ Res 2001;88:529-535.

20. Jennings RB, Steenbergen Jr C, Reimer KA. Myocardial ischemia and reperfusion. Monogr Pathol 1995;37:47-80.

21. Condorelli G, Drusco A, Stassi G, et al. Akt induces enhanced myocardial contractility and cell size in vivo in transgenic mice. Proc Natl Acad Sci USA 2002;99:12333-12338.

22. Chandraselar B, Mummidi S, Claycomb WC, et al. Interleukin-18 is a pro-hypertrophic cytokine that acts through a phosphatidylinositol 3-kinase-phosphoinositide-dependent kinase-1-Akt-GATA4 signalling pathway in cardiomyoctes. J Biol Chem 2005;280:4553-4567.

23. Schiekofer S, Shiojima I, Sato K, et al. Microarray analysis of Akt1 activation in transgenic mouse hearts reveals transcript expression profiles associated with compensatory hypertrophy and failure. Physio Genomics 2006;27:156-170.

24. Takano $H$, Zou $\mathrm{Y}$, Hasegawa $H$, et al. Oxidative stress-induced signal transduction pathways in cardiac myocytes: involvement of ROS in heart diseases. Antioxidant Redox Signal 2003;5:789-794.

25. Higuchi Y, Otsu K, Hirotani S, et al. Involvement of reactive oxygen species-mediated NF- $\kappa B$ activation in TNF- $\alpha$-induced cardiomyocyte hypertrophy. J Mol Cell Cardiol 2002;34:233-240.

26. Hosenpud JD, Bennett LE, Keck BM, et al. The registry of the international society for heart and lung transplantation: seventeenth official report: 2000. J Heart Lung Transplant 2000;19:909-931.

27. Bujak M, Frangogiannis NG. The role of TGF- $\beta$ signaling in myocardia infarction and cardiac remodeling. Cardiovasc Res 2007;74:184-195.

28. Furuyama A, Hirano $S$, Koike $E_{\text {, et }}$ al. Induction of oxidative stress and inhibition of plasminogen activator inhibitor-1 production in endothelial cells following exposure to organic extracts of diese exhaust particles and urban fine particles. Arch Toxicol 2006;80: 154-162.

29. Irani K, Xia Y, Zweier JL, et al. Mitogenic signaling mediated by oxidants in Ras-transformed fibroblasts. Science 1997;275:1649-1652.

30. Barcellos-Hoff MH, Dix TA. Redox-mediated activation of latent transforming growth factor- $\beta 1$. Mol Endocrinol 1996;10:1077-1083.

31. Miyazaki T, Karube M, Matsuzake $Y$, et al. Taurine inhibits oxidative damage and prevents fibrosis in carbon tetrachloride-induced hepatic fibrosis. J Hepatol 2005;43:117-125.

32. Pacher $P$, Schulz $R$, Liauder $L$, et al. Nitrosative stress and pharmacological modulation of heart failure. Trends Pharmacol Sci 2005;26:302-310.

33. Rakhit RD, Mojet MH, Marber MS, et al. Mitochondria as targets for nitric oxide-induced protection during simulated ischemia and reoxygenation in isolated neonatal cardiomyocytes. Circulation 2001;103:2617-2623.

34. Ishibashi $Y$, Shimada T, Sakane $T$, et al. Contribution of endogenous nitric oxide to basal vasomotor tone of peripheral vessels and plasma B-type natriuretic peptide levels in patients with congestive heart failure. J Am Coll Cardiol 2000;36:1605-1611.

35. Zhao T, Xi L, Chelliah J, et al. Inducible nitric oxide synthase mediates delayed myocardial protection induced by activation of adenosine $A(1)$ receptors: evidence from gene-knock-out mice. Circulation 2000;102:902-907.

36. Zweier JL, Samouilov A, Kuppusamy P. Non-enzymatic nitric oxide synthesis in biology systems. Biochim Biophys Acta 1999;1411: 250-262.

37. Bolli $\mathrm{R}$, Dawn $\mathrm{B}$, Tang $\mathrm{XL}$, et al. The nitric oxide hypothesis of late preconditioning. Basic Res Cardiol 1998;93:325-338.

38. Mannick JB, Asano K, Izumi K, et al. Nitric oxide produced by human $B$ lymphocytes inhibits apoptosis and Epstein-Barr virus reactivation. Cell 1994;79:1137-1146.

39. Imamura G, Bertelli AA, Bertelli A, et al. Pharmacological preconditioning with resveratrol: an insight with iNOS knockout mice. Am J Physiol Heart Circ Physiol 2002;282:H1996-H2003. 